INPLASY

PROTOCOL

To cite: Yang et al. Traditional Chinese mind-body exercises for insomnia: a protocol for systematic review and metaanalysis. Inplasy protocol 202150073. doi:

10.37766/inplasy2021.5.0073

Received: 18 May 2021

Published: 19 May 2021

Corresponding author:

Deyu Cong

congdeyu@sina.com

Author Affiliation:

Changchun University of

Chinese Medicine

Support: $2018 Y F c 1706006$.

Review Stage at time of this submission: Piloting of the study selection process.

Conflicts of interest:

None declared.

\section{Traditional Chinese mind-body exercises for insomnia: a protocol for systematic review and meta-analysis}

Yang, K1; Xie, DL2; Hu, GY3; Wang, YF4; Zhang, HS5; Lou, HJ6; Cong DY7.

Review question / Objective: Traditional Chinese mind-body exercises is a representative therapy for the treatment of insomnia, and its effectiveness and safety has been validated clinically. However, there is a lack of systematic evaluation and analysis of traditional Chinese mind-body exercises. Therefore, the effectiveness and safety of traditional Chinese mind-body exercises in the treatment of insomnia are studied and systematically evaluated in this study.

Condition being studied: Insomnia is a global public problem, which has a significant negative impact on both physical and mental health, while increasing the economic burden placed on both sufferers and society. Due to the side effects of pharmacological therapy, people are seeking to choose complementary and alternative therapies for insomnia disorder.

INPLASY registration number: This protocol was registered with the International Platform of Registered Systematic Review and Meta-Analysis Protocols (INPLASY) on 19 May 2021 and was last updated on 19 May 2021 (registration number INPLASY202150019).

\section{INTRODUCTION}

Review question / Objective: Traditional Chinese mind-body exercises is a representative therapy for the treatment of insomnia, and its effectiveness and safety has been validated clinically. However, there is a lack of systematic evaluation and analysis of traditional Chinese mind-body exercises. Therefore, the effectiveness and safety of traditional Chinese mind-body exercises in the treatment of insomnia are 
studied and systematically evaluated in this study.

Condition being studied: Insomnia is a global public problem, which has a significant negative impact on both physical and mental health, while increasing the economic burden placed on both sufferers and society. Due to the side effects of pharmacological therapy, people are seeking to choose complementary and alternative therapies for insomnia disorder.

\section{METHODS}

Search strategy: Two sets of search terms will be used, as follows: (1) "Chronic Insomnia" or"Primary Insomnia" or"Secondary Insomnia"or"Comorbid Insomnia"or"Disorder of Initiating and Maintaining Sleep"or"Behavioral Insomnia of Childhood "or"Sleep-onset Association Disorder"or"Limit-setting Sleep Disorder";and (2) "Tai Chi" or "Tai Chi Chuan" or "Qigong" or "Baduanjin" or "Wuqinxi" or "Yijinjing".

Participant or population: Studies will be eligible if they include participants with a diagnosis of insomnia.There were no limitations on age, gender, or nationality of patients with insomnia.

Intervention: Traditional Chinese mindbody exercises include Tai Chi, Qigong, Baduanjin, Wuqinxi, and Yijinjing. The included studies use one of them to treat insomnia. The control interventions include medicine, observation, manual therapy, acupuncture, traction, education, and any treatments without traditional Chinese mind-body exercises. The study assessing the effects of traditional Chinese mindbody exercises plus an intervention compared with the same intervention (such as Tai Chi plus traction versus traction) will also be included.

Comparator: The included studies use one of them to treat insomnia. The control interventions include medicine, observation, manual therapy, acupuncture, traction, education, and any treatments without traditional Chinese mind-body exercises.

Study designs to be included: This review will only include RCTs of traditional Chinese mind-body exercises for insomnia. Case reports, observational studies, and cross-sectional design studies will be excluded. The study protocol and conference abstract of RCTs will also be excluded, while the corresponding author could not provide detailed information.

Eligibility criteria: Two experienced reviewers will independently search all databases according to the search strategies, read the titles and abstracts to select potential references, exclude obviously unrelated literature, and delete duplicated relevant research abstracts. Then, they will read the full text to assess eligible studies. If the information is incomplete, the reviewer should contact the author to obtain complete information. If there is a disagreement it will be resolved through discussion or a third reviewer.

Information sources: The electronic databases (PubMed, Embase, Web of Science, Cochrane Library, China Knowledge Resource Integrated Database, and Wanfang Data) will be searched from their inception to June 2021.

Main outcome(s): The primary outcome includes Pittsburgh sleep quality index.

\section{Additional outcome(s): Insomnia Severity Index.}

Data management: The quality assessment of the included studies will be independently conducted by two reviewers using the Physiotherapy Evidence Database (PEDro) scale. The PEDro scale is a tool developed to measure the methodological quality of RCTs of physiotherapy interventions.[23, 24] The scale involves an 11-domain assessment: 1) study eligibility criteria specified, 2) random allocation of subjects, 3) concealed allocation, 4) measure of similarity between groups at baseline, 5) subject blinding, 6) therapist blinding, 7) 
assessor blinding, 8) less than $15 \%$ dropouts, 9) intention- to-treat analysis, 10) between-group statistical comparisons, and 11) point measures and variability data. The PEDro score will be calculated by criteria 2) to 11) according to meeting the criteria or not. From these scores, the studies are considered as excellent (9-10 points), good (6-8 points), fair (4-5 points), and poor (less than 4 points) quality.

Quality assessment / Risk of bias analysis: According to the recommendations of the Cochrane handbook for systematic reviews of interventions, the heterogeneity will be assessed using the statistic and $Q$ statistic. Three levels of heterogeneity are $25 \%$ (low heterogeneity), $50 \% \quad$ (moderate heterogeneity) and $75 \%$ (high heterogeneity). It will be considered to be statistically significant when P0.10. An Egger's test was performed to examine publication bias, and publication bias will be determined from a corresponding $p$ value less than 0.05 .

Strategy of data synthesis: The metaanalysis will be conducted using Review Manager Version 5.3 software. For continuous data, the change between baseline and the end of interventions will be used in the meta-analysis. The mean difference (MD) and $95 \%$ confidence intervals $(\mathrm{Cl})$ will be calculated. In the case of different outcome measure scales, the standardized mean difference (SMD) and 95\% Cl will be calculated. For the expected heterogeneity, the continuous data will be pooled using a more conservative randomeffects model.

Subgroup analysis: If considerable heterogeneity is found, subgroup analysis would be envisaged to perform to investigate probable sources of heterogeneity in accordance with the duration of treatment, age, history of insomnia, and research quality.

Sensitivity analysis: In order to ascertain the sensitiveness of results to modifications in initial assumptions, the authors will conduct sensitivity analysis by only including studies with low risk of bias.
Language: English.

Country(ies) involved: China.

Keywords: traditional Chinese mind-body exercises, insomnia, meta-analysis, systematic review.

Contributions of each author:

Author 1 - Kang Yang.

Email: yangkang5537@163.com

Author 2 - Dongling Xie.

Email: 1021586903@qq.com

Author 3 - Guanyu Hu.

Email: 1241453233@qq.com

Author 4 - Yufeng Wang.

Email: wangchn@126.com

Author 5 - Hongshi Zhang.

Email: 5503576@qq.com

Author 6 - Huijuan Lou.

Email: 1240740144@qq.com

Author 7 - Deyu Cong.

Email: congdeyu666@sina.com 